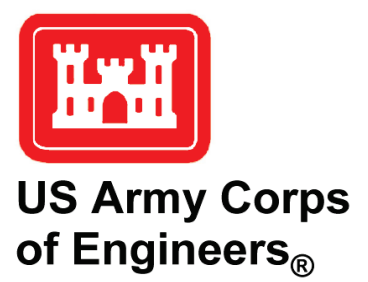

\section{Development of a Guided-Wave Technology Capable of the Detection of Open Cracks and Microcracks in Embedded Trunnion Anchor Rods}

by James A. Evans and Rick Haskins

BACKGROUND: Catastrophic failure of post-tension trunnion anchor rods at dams is an ongoing problem caused by propagating orthogonal stress corrosion cracks described in CHETN-IX-32 (Evans et al. 2013). This condition is shown in Figure 1 which presents a cracked rod that was removed from Robert F. Henry Lock and Dam, which has 376 trunnion rods supporting the tainter gates. In the broken rod image, a blue arrow indicates the fracture origin, and white arrows indicate the crack propagating direction. The green ellipse encircles an area that contains a pre-existing crack. This older crack area is more heavily oxidized than the rest of the fracture surface. This crack over time resulted in the complete failure of the rod. The right side of Figure 1 shows the tainter gate trunnion rods located on a dam pier. These rods serve to prestress the concrete to prevent excessive tensile loading on the concrete during gate operation.
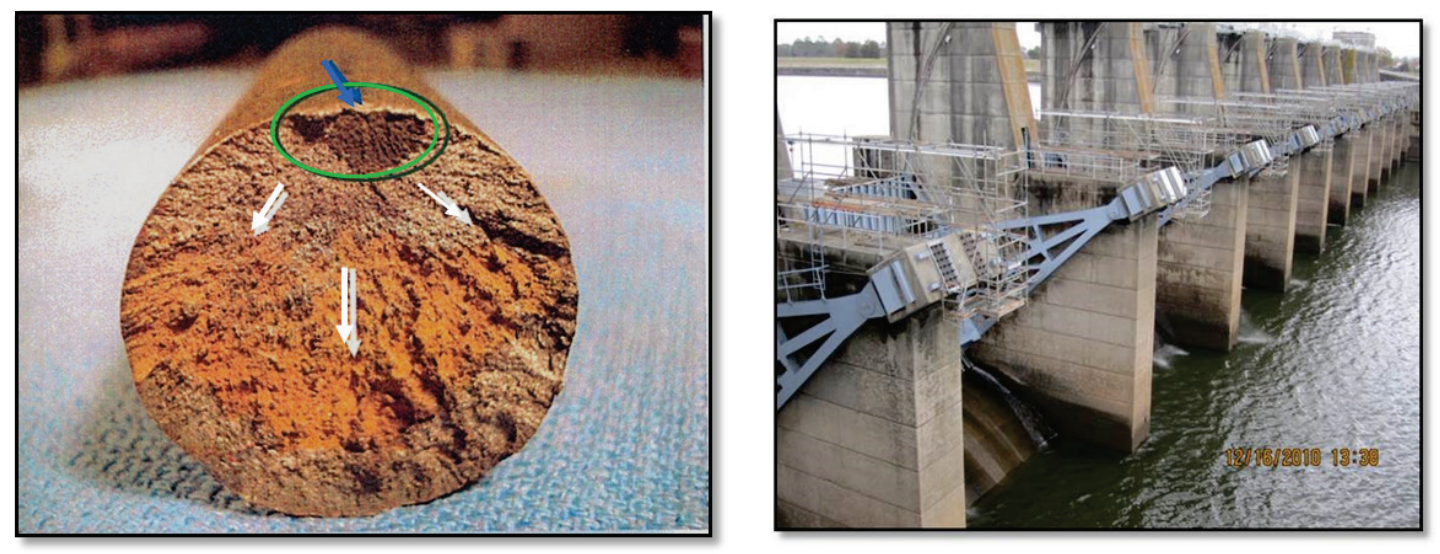

Figure 1. Cracked trunnion rod from Robert F. Henry Lock and Dam

In addition, CHETN-IX-32 provides background information and initial test-bed investigations of nonlinear and guided-wave methods directed at development of a non-destructive testing capability for long-distance microcrack detection in embedded trunnion rods. A second technical note (Evans and Haskins, forthcoming) describes performance of an initial prototype guidedwave system in field testing that was performed at Greenup Lock and Dam.

The second document also encompasses US Army Engineer Research and Development Center (ERDC) test-bed characterization of hardware improvements and measurement influences that were made following the Greenup Lock and Dam testing. 
PURPOSE: The purpose of this document is to provide additional background and performance information regarding the use of higher-order longitudinal modes for long-range, embedded-rod inspection. Rod and system parameters and their influence on defect detection, particularly those not covered in CHETN-IX-32, will be addressed as well as a preliminary description of a guidedwave testing process suitable for open-crack detection of long, embedded steel rods.

\section{APPROACH:}

Higher-Order Longitudinal Modes. The field boundary constraints of remotely detecting defects in an embedded rod with access limited to one end makes conventional ultrasonic nondestructive testing (NDT) methods a poor fit. Guided-wave acoustics provides an NDT solution for remote detection in an embedded symmetrical body when only one end of the rod is accessible. If these cracks are closed and hence poor reflectors, then nonlinear methods must be incorporated under the constraints imposed by guided-wave propagation in the element (Pruell 2009; Jhang 2000). A number of investigators have covered rod-guided waves with the intent of solving problems such as rebar corrosion in concrete, rock anchor-bolt bonding, and utility pole, guidewire anchor corrosion in soils (Beard 2003; Erwin et al. 2009; Yang and Yu 2006; He et al. 2006 ). In general, these three specific applications need high sensitivity at the rod surface and therefore benefit from narrow-band, guided-wave modes such as low-order longitudinal or torsional modes that result in relatively higher levels of acoustical energy at or near the rod's surface. The drawback of these lower-frequency modes is that energy leaks into the surrounding medium at a higher rate producing correspondingly higher attenuation rates and therefore reducing the maximum testable rod length. Low-order attenuation cannot be overcome by simply continuing to apply more power because piezoceramic materials are limited by their dielectric breakdowns and/or depoling at extremely high voltage levels.

The trunnion anchor rod has an additional energy leak concern as the rods are typically 0.125 in. or closer to the steel sleeve that contains the rod and its corrosion protection media of grease or grout. Test-bed experiments confirm that frequencies between 12 and $24 \mathrm{kHz}$ tend to travel through the grease and propagate back and forth on the schedule 40 pipe. Propagation down these sleeves and their couplers or reflections from random rod/sleeve contact points would be a potential false-alarm source if low-order longitudinal modes were pursued for inspection of embedded trunnion anchor rods.

Testing long lengths of embedded rods with vertical or horizontal shear modes is likewise problematic as these modes produce higher leakage into the surrounding medium and additionally suffer from higher material-attenuation rates. Pavlakovic et al. (2001) provide an excellent history of guided-wave theory development for rods and further demonstrate that for long-distance inspection, higher-order longitudinal modes are the best solution. Energy becomes increasingly centralized in the rod, and leakage rates at the surface are reduced proportionally. As frequency continues to increase, material-attenuation rates also increase, so a point of diminishing return is encountered. Figure 2 illustrates the principles behind the optimal higher-order, low-loss propagation modes. The increased energy centralization that occurs at higher frequencies (i.e., higher longitudinal modes) minimizes surface displacements and energy leakage into the surrounding medium (Ervin 2009). As previously stated, the limitation of what can be gained from energy centralization occurs at higher frequencies where the material attenuation begins to impede 
ultrasonic propagation. An example spectral range of low-loss longitudinal modes in a 1.125 in. bar is shown below the two superimposed gain curves in Figure 2.

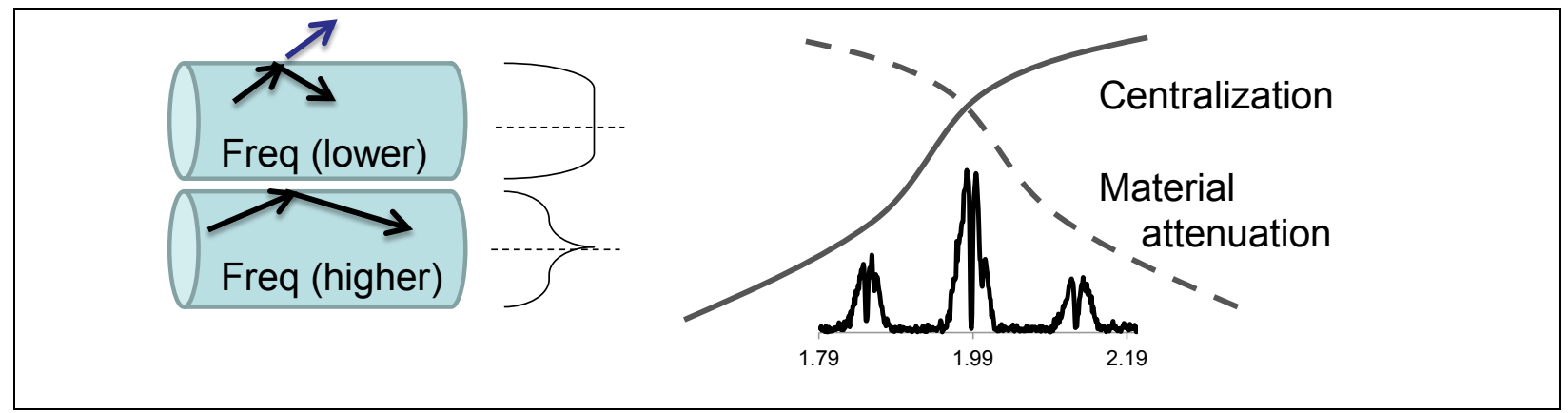

Figure 2. Low-loss modes existing between centralization of guided-wave energy at higher frequency and lower material attenuation at lower frequencies.

Figure 3 illustrates an experimentally collected, broad-band energy velocity curve for various embedded rod modes in (a) and the higher frequency and sustained modes in (b) that were captured in multiple reverberations. These higher-frequency modes in the 1 to $3 \mathrm{MHz}$ range are the most practical for long-distance trunnion anchor rod testing. The time-frequency plot's A-Scan is shown above the spectrogram in (b) and shows the roughly $2 \mathrm{MHz}$ range of modes undergoing low attenuation through two reverberations.

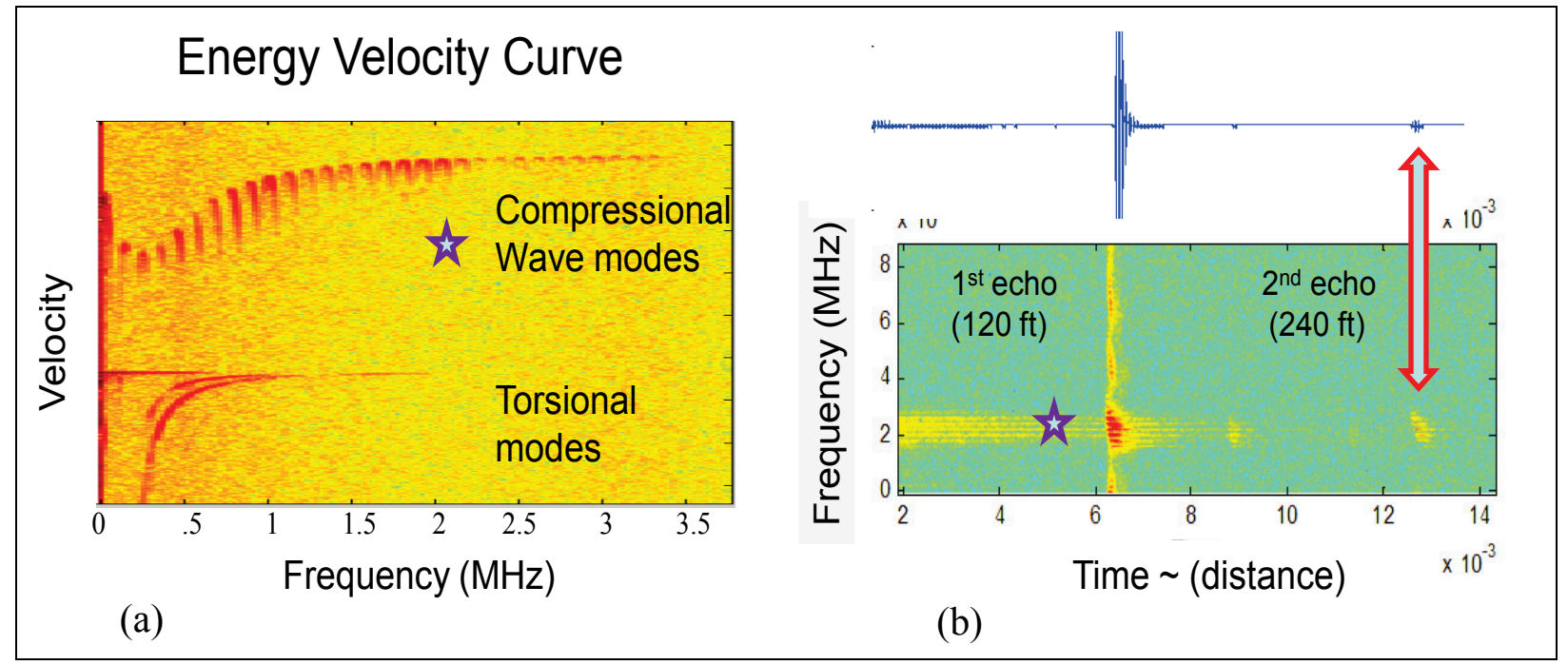

Figure 3. (a) Energy velocity curves showing compression and torsional modes; (b) Reverberation of high-frequency, low-loss modes and the associated A-scan.

To evaluate the capabilities for shallow, near-surface defect detection, an experiment was performed where a progressively deepening cut was made while monitoring the expected reflection location in the ultrasonic A-scan. This cut notch was first detected with an approximate signal-tonoise ratio of 2 using the low-loss, narrow-band inspection frequency at a depth of $0.1 \mathrm{in}$. at a distance 17 feet from the free end of a grease-embedded 1.2 in. diameter rod (Figure 4). As the cut was increased, the echo amplitude grew as expected at a very high rate because of the increased amount of material removed from the rod's cross-section and the reflection of the higher energy 
concentrations as the cut plane migrated towards the rod's center where the energy is more centralized. The spectral signature of the cut (i.e., open crack) can also serve as a signature for in situ monitoring purposes.

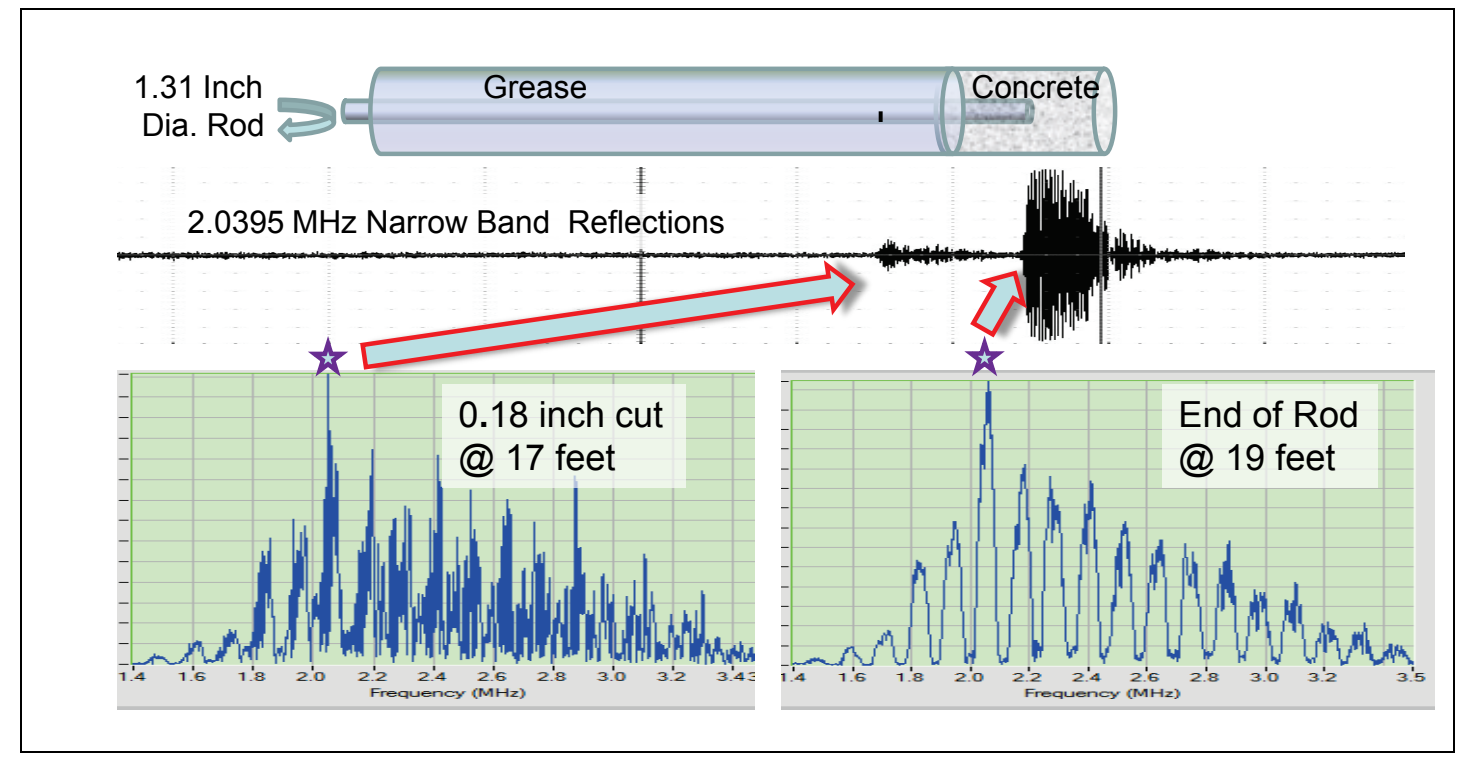

Figure 4. Narrow-band spectral reflections of a $0.18 \mathrm{in}$. deep cut and the rod end of a 1.31 in. diameter, grease-embedded trunnion anchor rod with concrete termination.

Physical Propagation Influences. Rod diameter has a significant influence on the ultrasonic attenuation rates as well as the frequency spacing and location of the low-loss modes. Comparison of rod diameters that are 1.25 and 1.125 in. results in an attenuation rate increase by a factor of 3 for the smaller diameter rod. According to EM-1110-2-2702 (Headquarters, USACE 2000), a 1.0 in. rod is the minimal allowable rod diameter for tainter gate anchorages. Sevenstrand cables are also used at some dams. At present, cable inspection lengths are limited to 12.5 $\mathrm{ft}$ at a low-loss mode approximately $5 \mathrm{MHz}$. In its current state, the ERDC guided-wave system can inspect the more attenuative 1.125 in., grease-embedded anchor rods at lengths exceeding $100 \mathrm{ft}$. Because of the low-surface leakage, the grout embedding medium results in only a minimal increase in attenuation when compared to grease embedment. This has been verified in test-bed experiments (Beard and Lowe 2003). A high-power/high-sensitivity piezoelectric material, manganese-doped lead indium niobate-lead magnesium niobate-lead titanate (Mn PINPMN-PT) (Luo et al. 2009) is currently being acquired for evaluation. This new piezoelectric material is reported to potentially produce gains up to a factor of 8 over commercially available, high-performance PZT materials.

Experimentation has demonstrated that rod tension and corresponding bulkhead loading have a negligible influence on the propagation of high-frequency, low-loss modes. Other physical rod characteristics, however, should also be considered as their influence can be quite significant. Rod-end condition is a significant influence and can vary in terms of both angle and smoothness. In conventional field installations, the far anchored bulkhead is typically threaded and therefore not typically cut. Threaded rods can have some small surface variations, but these tend to be symmetrical and consistent from rod to rod and therefore are taken care of in the initial scanning 
for inspection frequency (this is discussed in more detail later). Because of this, the cut angle and rod smoothness are more of an issue on the free, exposed end. If the rods are cut flat but at a nonorthogonal angle, significant losses can be occurred. Published data (Beard and Lowe 2003) and ERDC test-bed experimentation indicate that angles up to 10 degrees can produce attenuation rates up to $40 \mathrm{~dB}$ per reverberation. This is due to destructive phase interference and mode conversion of the propagating guided waves. Additionally, surface roughness prevents efficient coupling of the transducer face and therefore impedes power transfer along with potential phase interference if roughness is on the order of a quarter wavelength. A number of techniques are being investigated to either produce the optimum surface on the rod's end or to compensate for its roughness via either high impedance fillers or alternate transduction schemes. These results will be discussed in the final project report; however, a tentative scheme for quickly coupling to rough-ended rods is discussed below.

An experiment was performed to determine if gallium, which has an acoustical impedance of approximately 17-20 MRayls and transitions from solid to liquid at very low temperatures, could be effectively used to couple ultrasonic energy from the transducer to a rough-ended (torch-cut) trunnion rod. The gallium, which quickly melted in a hot water bath, was poured into a mold attached to the rod end. Ice was used to speed the transition back to a solid state, which took approximately 5 to 10 minutes. Before ultrasonic evaluation, a small layer of grease was applied between the rod end and the cast gallium piece (Figure 5, top left). Pitch-catch measurements and through-transmission readings were made by grease coupling and clamping the transducer to the smooth gallium face (Figure 5, bottom left). The A-scan in Figure 5 (right) shows the reverberations observed in a very short section of trunnion anchor rod that had been field cut with a torch. The results from this experiment are encouraging and indicate that gallium casting to rough ended rods might provide a rapid and safe alternative to cutting, sanding, and other finishing approaches (Culjat et al. 2005). Future investigations will look at long, embedded-rod sections to further evaluate the effectiveness of this coupling strategy and also to characterize the influence of the irregular surface interface on guided-wave inspection. A worst-case scenario is that frequency scans may be needed on each rough-cut rod.

Generally, in-service trunnion anchor rods should be straight due to their high level of tension. If the majority of applied tension has been lost or if the rod is deflected due to poor sleeve alignment of the grout/grease tube, then deflection of the rod might occur. Published guidedwave research indicates that rod curvatures on the order of 800 times the rod's radius can be detected via phase interference on propagating guided waves (Christoph et al. 2009). Test-bed experiments have verified that phase interference from rod curvature is detectable via spectral changes in the low-loss longitudinal modes; however, significant deflection was required for the effect to become detectable in the propagating low-loss modes.

HARDWARE DESIGN: Even with site-specific, low-loss mode propagation modes, detection of reflected echoes through hundreds of feet of embedded rod is very system demanding and requires exploitation of multiple enabling methods and technologies. Table 1 provides information that describes various mechanisms in the current ERDC system and their contribution toward improved system performance. 

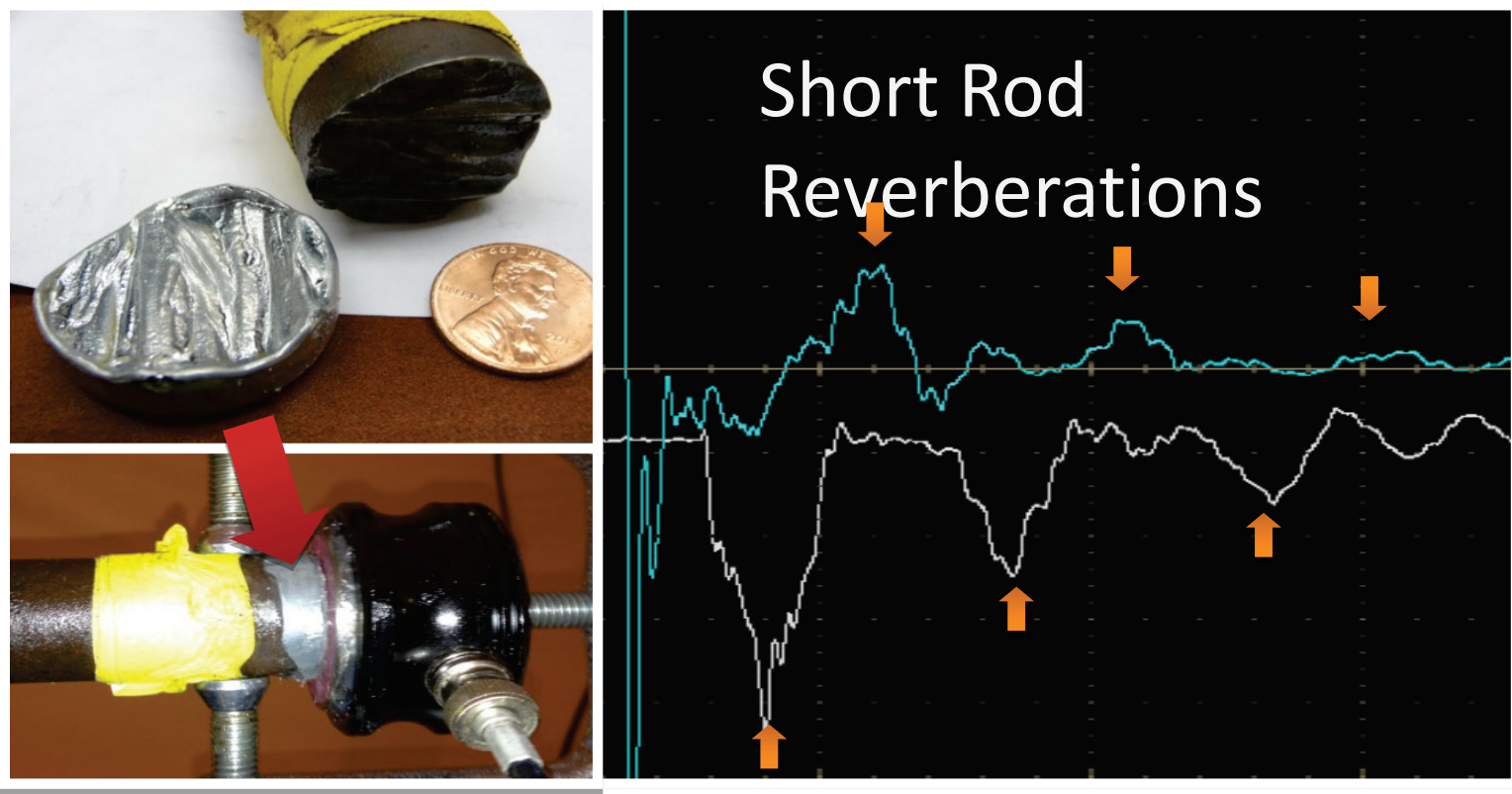

Figure 5. Gallium used as interface between rod and piezoelectric transducer.

\begin{tabular}{||l|l||}
\hline \multicolumn{2}{|l|}{ Table 1. Current guided-wave system and improvements to be made. } \\
\hline \hline System Component or Method & Benefit or Contribution \\
\hline Signal Averaging & $\begin{array}{l}\text { Reduction in non-stationary noise which is largely } \\
\text { due to the high system gain ( 100 db). }\end{array}$ \\
\hline Narrow-band signal (70-200 cycles) & $\begin{array}{l}\text { Concentrates energy in desired propagation mode } \\
\text { and improves response of PZT transducer. }\end{array}$ \\
\hline High output/sensitivity PZT or PMN-PT & Maximum performance transduction technologies. \\
\hline Time-corrected gain amplifier with nonlinear ramp & $\begin{array}{l}\text { Extends detectability to entire rod length while still } \\
\text { allowing high gain and high pulser voltages. } \\
\text { Increases dynamic range of system. }\end{array}$ \\
\hline Large-area/medium-damped transducer & $\begin{array}{l}\text { Maintains high output levels and sensitivity while } \\
\text { eliminating undesired transducer reverberations } \\
\text { experienced with low-damped (high-response) } \\
\text { transducers. }\end{array}$ \\
\hline High-power pulser (5,000 Watts) & $\begin{array}{l}\text { Stimulates transducer with maximum allowable } \\
\text { voltage. Must be stimulated below piezoceramic } \\
\text { failure levels. }\end{array}$ \\
\hline Superhetrodyne phase detector & $\begin{array}{l}\text { Improved detection of narrowband signal amplitude } \\
\text { and phase information in the presence of stationary } \\
\text { noise. Demodulates RF. }\end{array}$ \\
\hline Very fast recovery preamplifier & $\begin{array}{l}\text { Prevents preamplifier saturation of diplexer output } \\
\text { from masking rod defects that may be located near } \\
\text { the transducer. }\end{array}$ \\
\hline High-pass and low-pass filtering & $\begin{array}{l}\text { Reduction of unwanted noise sources. } \\
\text { Optimizes pitch-catch operation and takes } \\
\text { advantage or limited injection area. }\end{array}$ \\
\hline Tunable diplexer & $\begin{array}{l}\text { Allows determination of optimum low-loss } \\
\text { inspection modes at each site. }\end{array}$ \\
\hline narrowband signals &
\end{tabular}


The current system can acquire over $600 \mathrm{ft}$ of propagation length on $1.25 \mathrm{in}$. diameter rods. As a result, multiple backwall echoes can often be acquired. This is a desirable configuration as it allows improved confidence with the second and third occurrence of any defect echoes. Notably, some small spectral changes occur due to the influence of the transducer absorbing and therefore slightly changing the spectral content of what is reverberating in the rod. This effect is constant and only changes the received spectrum between the first and second reverberation.

Test Method of Current System. An optimized system hardware configuration detailing the relative location of the various components was determined following the Greenup Lock and Dam testing and presented in the technical note Guided Wave Testing of Trunnion Rods at Greenup Dam, Kentucky (in preparation). The current process used for the optimization of system operational parameters forms the beginning of a test method for open-crack detection. In its current state the overall method for testing at a new site is as follows: (1) Inspect rod-end conditions and perform quick-tap test survey. If rods need end dressing, use portable band saw and a belt sander along with a removable, steel guide collar to achieve a smooth orthogonal end condition or perhaps use cast gallium coupling pieces for torch-cut ends. (2) Perform frequency scanning of the end-ofrod echo gate from approximately 1 to $3 \mathrm{MHz}$ with the transducer clamped to rod end to determine optimum low-loss propagation modes. This should be performed on several rods within each anchoring group to assure consistency. (3) After determining an optimum low-loss mode from the frequency scan, set up the time-corrected gain circuit, signal averaging, pulse cycle count, and diplexer options to acquire optimal echo response. (4) Begin signal collection using the determined system settings. (5) If a defect echo is received (i.e., ahead of the back wall), perform a gated frequency scan of the back wall and new echo is received by setting appropriate gate positions. (6) If the echo exhibits maximal response at other than the predetermined scanning frequency or frequencies, then recollect a new A-scan at this maximal response for later analysis. (7) Resume inspection of remaining rods using the frequency or frequencies acquired at step 4.

Embedded System Requirements. Since the test method just presented is for configuration of a laboratory type system, it is more involved than that of a specialized, embedded system. The embedded system will simplify several configuration steps such as frequency scanning, optimal cycle count determination, and the setting of the time-corrected gain function. Additionally, the requirements for detection of nonlinear influences will also be incorporated and require other components of inspection and setup. These might be simple, with nonlinear methods such as frequency mixing or harmonic analysis, or somewhat more involved for methods such as impact modulation.

Migration from the laboratory system to an embedded system is needed for several reasons. In addition to simplicity of setup and operation as mentioned above, the portability and cost can further be improved. If permanent on-site, long-term monitoring is desired, the system size and cost parameters become even more critical. Initially the option of modifying or reconfiguring existing commercial non-destructive testing (NDT) hardware was considered as it would provide the most cost-effective transition strategy. The systems investigated for this purpose included sonar, metal inspection, guided-wave pipe systems, and more specialized airborne composite systems. These systems failed to be adaptable for a multitude of reasons.

The general hardware configuration currently used is a 2 in. $2.25 \mathrm{MHz}$ Accuscan transducer, A Ritec Instruments ultrasonic RAM-5000 SNAP (http://www.ritecinc.com/snapspecs.pdf) system 
with linear diplexer and fast recovery preamplifier, along with a high-end oscilloscope and a function generator driving a time-corrected gain circuit. Better or equal performance using a reduced hardware footprint is the embedded-system transition goal. An initial step will be to integrate the fast recovery preamplifier with the time-corrected gain circuit and its driving function generator. The second phase will be to transition from the high-end digital storage oscilloscope to either a notebook computer or computer on a stick (http://www.laptopmag.com/androidsticks.aspx) interfacing a high-performance, low-cost digitizer such as the PicoScope 5244B (http://www.pc-oscilloscopes.com/oscilloscope-chart.html). The final stage will be focused on replacement of the Ritec system's pulse generation and, depending on their necessity, its heterodyne-detection and nonlinear-measurement capabilities. Texas Instruments is an example of a vendor that supplies numerous specialized integrated circuits that are used for developers of embedded ultrasonic systems and whose component offerings may be useful in this hardware transition phase (http://www.ti.com/corp/docs/landing/afe5805/index-google.htm?DCMP=PPC Google TI\&k_clickid=3abe6108-0687-3be8-72b4-00000a41a92d\&247SEM=).

SUMMARY: The USACE required the use of post-tensioned trunnion anchor rods in the design of spillway tainter gates in the 1960s and 1970s. During this period, several navigation, flood control, and hydroelectric projects were completed. These post-tensioned trunnion anchor rods were used extensively for support of tainter gates and considered the standard for the USACE and other government and nongovernment agencies within the United States and elsewhere. Post-tensioned rods are used to anchor spillway gates and transfer the forces from the reservoir pool through the gates to the spillway structures. Large tensile loads are applied to these high-strength steel rods to compress the surrounding concrete and prevent it from experiencing excessive tensile forces, which are naturally problematic for concrete. These rods are now experiencing ongoing failures, and the Corps of Engineers is developing a reliable non-destructive testing (NDT) method that is rapid, robust, and capable of detecting and quantifying trunnion rod defects. ERDC engineers are in the process of developing a procedure and equipment using guided acoustical waves and nonlinear acoustics to detect and quantify defects in these rods.

ADDITIONAL INFORMATION: This CHETN is a product of the NavSys Program of the Navigation Safety Research Program being conducted at the US Army Engineer Research and Development Center, Coastal and Hydraulics Laboratory. Questions about this technical note may be addressed to James Evans: 601-634-2535; email: james.a.evans@usace.army.mil. This technical note should be cited as follows:

Evans, J. A., and R. W. Haskins. 2014. Development of a guided-wave technology capable of the detection of open cracks and microcracks in embedded trunnion anchor rods. ERDC/CHL CHETN-IX-36. Vicksburg, MS: US Army Engineer Research and Development Center. http://chl.erdc.usace.army.mil/chetn

\section{REFERENCES}

Beard M. D., and M. J. S. Lowe. 2003. Non-destructive testing of rock bolts using guided ultrasonic waves. London, United Kingdom: Department of Mechanical Engineering, Imperial College.

Christoph, P., Q-Y Kim, J. Qu, and L. J. Jacobs. 2009. Evaluation of fatigue damage using non-linear guided waves. Atlanta, GA: School of Civil and Environmental Engineering, Georgia Institute of Technology. 
Culjat M. O., R. S. Singh, S. N. White, R. R. Neugonkar, and E. R. Brown. 2005. Evaluation of gallium-indium alloy as an acoustic couplant for high-impedance, high-frequency applications. Acoustics Research Letters Online, 10 June 2005. Acoustical Society of America. http://scitation.aip.org/content/asa/journal/arlo/ $\underline{6 / 3 / 10.1121 / 1.1903025}$

Ervin, B. L., D. A. Kuchma, J. T. Bernhard, and H. Reis. 2009. Monitoring corrosion of rebar embedded in mortar using high-frequency guided ultrasonic waves. J. Eng. Mech. 135(1):9-19.

Evans, J. A., R. W. Haskins, J. A. Padula, and J. E. Hite. 2013. Trunnion rod microcrack detection. ERDC/CHL CHETN-IX-32. Vicksburg, MS: US Army Engineer Research and Development Center.

Evans, J. A., and R. W. Haskins. (Forthcoming). Guided wave testing of trunnion rods at Greenup Dam, Kentucky. Vicksburg, MS: US Army Engineer Research and Development Center.

He, C., J. K. Van Velsor, C. M. Lee, and J. L. Rose. 2006. Health monitoring of rock bolts using ultrasonic guided waves. P. R. China: Beijing University of Technology; Park, PA: The Pennsylvania State University.

Headquarters, US Army Corps of Engineers (USACE). 2000. Design of spillway tainter gates. EM-1110-2-2702. Washington, DC: US Army Corps of Engineers.

Jhang, K-Y. 2000. Applications of non-linear ultrasonics to the NDE of material degradations. Seoul, Korea: School of Mechanical Engineering, Hanyang University.

Luo, J., W. Hackenberger, S. Zhang, and T. R. Shrout. 2009. The progress update of relaxor piezoelectric single crystals. Ultrasonics Symposium (IUS), 2009 IEEE International.

Pavlakovic, B. N., M. J. S. Lowe, and P. Crawley. 2001. High-frequency low-loss ultrasonic modes in imbedded bars. London, United Kingdom: Department of Mechanical Engineering, Imperial College.

Pruell, C., J. Y. Kim, J. Qu, and L. Jacobs. 2009. Evaluation of fatigue damage using nonlinear guided waves. Smart Materials and Structures 18:1-8.

Yang, H., and L. Yu. 2006. Inspected simulation of port anchor metal rods ultrasonic guide wave based on dispersion characteristics. Taiyuan, China: National Key Laboratory for Electronic Measurement Technology, North University of China (NUC).

NOTE: The contents of this technical note are not to be used for advertising, publication,

or promotional purposes. Citation of trade names does not constitute an official endorsement or approval of the use of such products. 